\title{
Molecular Electronics - Devices and Circuits Technology
}

\author{
Paul Franzon, David Nackashi, Christian Amsinck, Neil \\ DiSpigna, Sachin Sonkusale \\ Department of Electrical and Computer Engineering \\ North Carolina State University, Raleigh, NC, USA \\ paulf@ncsu.edu
}

\begin{abstract}
Molecular electronics holds significant potential to outscale bulk electronic devices. However, practical issues have limited that potential to date. This paper reviews the function and design of molecular electronics and evaluates results to date in a circuits context.
\end{abstract}

\section{Introduction}

Molecular electronics has several potential advantages for being of interest as an electronic element. It has small size, typically on the range of a few nm, well below the total size projected for any FET. A second advantage is that molecules can self assemble onto surfaces, a very low-cost process. Their third advantage is that they can be designed at the atomic level, a feat not possible with bulk devices. Atomic level design permits a wide range of devices to be investigated, and potentially leads to precise control of electronic properties. For example, switching between isomers of the same chemistry should lead to radically different device properties.

This paper presents a two-level overview of molecular electronics. Section 2 focuses on device physics and understanding, while Section 3 evaluates some of these devices within a circuit's context.

\section{Molecular Devices}

Since the first suggestion that molecular elements could be designed to control electronic properties in a circuit [1], the vast majority of research in molecular electronics has focused on measuring and predicting electronic transport through organic devices. Organic materials of all types have been studied, including metallic and semiconducting carbon nanotubes, silicon nanowires, oligo(phenylene ethnylene) (OPE) based bistable molecular switches, insulating alkanethiol chains, 
slightly more conductive OPEs and oligo(phenylene vinylene)s (OPVs), and chargestorage molecular systems such as ferrocenes. Understanding electron transport and charge storage is extremely important to advance the process of engineering molecules for specific applications.

Where silicon device characteristics are engineered by varying the carrier density through doping techniques, designing molecular devices involves modifying electronic wavefunctions at a metal-molecule-metal junction [2] However, as silicon devices continue to shrink, the current modeling techniques become less accurate as the channel lengths no longer exhibit bulk properties [3]. This has resulted in a great deal of harmony between the fields of silicon nanoelectronics and molecular electronics, with each group leveraging off the knowledge created by the other.

Most of the molecular electronic compounds listed above are only just a few angstroms to tens of angstroms in length. With only a few atoms involved in electron transfer, the notion of a density of states becomes less accurate and the properties of these molecules are better described by the location and energy gaps of their highest occupied molecular orbital (HOMO) and lowest unoccupied molecular orbital (LUMO). With a few exceptions such as metallic and semiconducting carbon nanotubes, it is expected that the Fermi levels of the metallic contacts will lie within the HOMO-LUMO gap of most molecules. This is illustrated in Figure 1, where the energy gap within most molecules is likely to be approximately $2-3 \mathrm{eV}$.

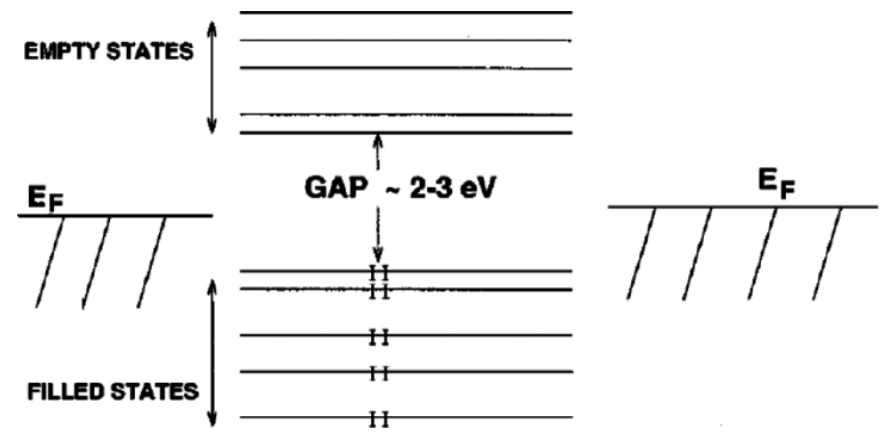

Fig. 1. Metal-Molecule-Metal junction. From Samanta et al. [4], "Electronic conduction through organic molecules."

With this model, it is expected that the primary mode of electron transfer will be tunneling, rather than propagation. A simple approximation for tunneling current through a molecular junction can be modeled using the expression, $\mathrm{k}_{\mathrm{ET}}=\mathrm{k}_{0} \mathrm{e}^{-\beta \mathrm{d}}$, where $\mathrm{k}_{\mathrm{ET}}$ is the rate of electron transfer, $\mathrm{d}$ is the barrier width (length of the molecule), and $\beta$ is a constant defined by the electronic structure of the organic layer. This approximation simply states that for set of similar molecules varying in length (such as alkanethiols with varying numbers of methylene groups), the current density (at a given voltage) across the junction will exponentially decrease as the molecular length increases. This is seen quite clearly in alkane chain conductivity research using mercury drop electrodes, nanopores and STM analysis. However comparing 
two structurally different molecules whose lengths are the same, research has shown that the tunneling currents can be very different. This has lead to the belief that the parameter $\beta$ can be used to describe the electron transmission properties of different molecules.

Experimental research has shown that molecules exhibiting a highly $\pi$ conjugated structure (such as OPEs and OPVs), as compared to the $\sigma$-bonded alkane chains, have a much lower gap resistance in metal-molecule-metal structures. This suggests that the barrier to electron transfer is lowered within molecules containing delocalized electron clouds. To more accurately account for scattering (which is neglected in the tunneling approximation) and the specific electronic structure for various molecules, many theorists use Density Functional and Green's Function based approaches for more accurate evaluations. An example of the value in these approaches is shown in Figure 2, where the transmission properties for three different molecules were calculated and plotted. Samanta and Datta [4] found that the resistance of a four benzene chain molecule scaled higher as expected when compared to a shorter, three benzene molecule. However, a three ring OPE was found to have a lower resistance than the four ring benzene chain, even though the OPE is a longer molecule. This was attributed to the presence of the triple bond in the OPE, causing a more delocalized electronic structure.

In the same study, Samanta calculated the transmission properties of a two ring benzene chain as a function of the ring orientation to each other. Shown in Figure 3, the most conductive state is when the molecule has no offset, or

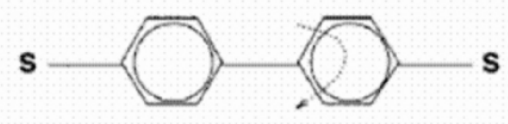

ANGLE BETWEEN TWO RINGS $=\theta$

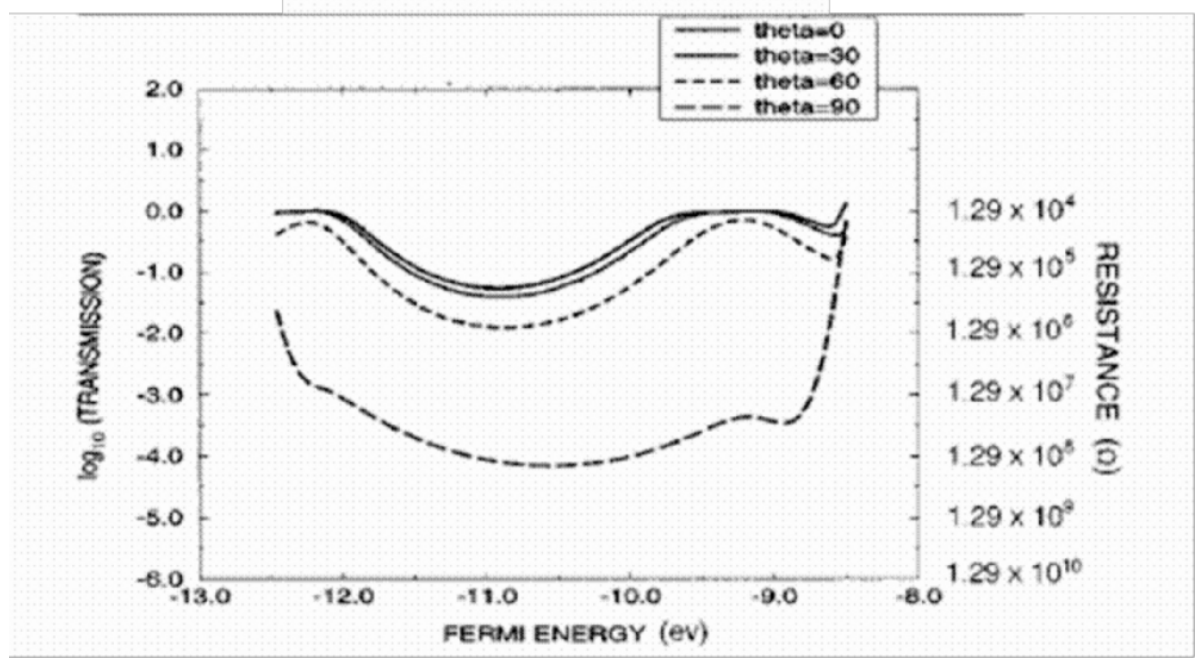

Fig. 2. Green-function analysis of electronic transmission through different molecules. From Samanta, et al. [4], "Electronic conduction through organic molecules." 
is planar, and the least conductive state is when the rings are $90^{\circ}$ out of phase. This theoretical work further suggests that delocalized, overlapping $\mathrm{p}$ orbitals play an import part in lowering the barrier for electronic conduction. Experimentally, this was also shown in two separate test structures comparing OPE and OPV molecules. OPV molecules, known to be more planar and exhibiting less bond-length alternation, were determined to have a slightly lower gap resistance.

Many have suggested using a third gate electrode to modulate transmission properties through a molecule by twisting or bending the molecular backbone, however concentrating a strong enough field in a gap less than 50 angstroms is extremely difficult. A theoretical study of a molecular three terminal device was performed by Datta ${ }^{\mathrm{i}}$ at Purdue, using a single benzene ring as the conductive channel. To get good control of the channel, i.e., to get a high enough field to modulate the device, the equivalent gate oxide would need to be less than $10 \%$ of the channel length. This suggests that the gate electrode would need to be within two angstroms of the benzene ring, placed within an atomic level of accuracy. For these reasons, most research into molecular electronics has focused on two terminal devices, primarily switches.

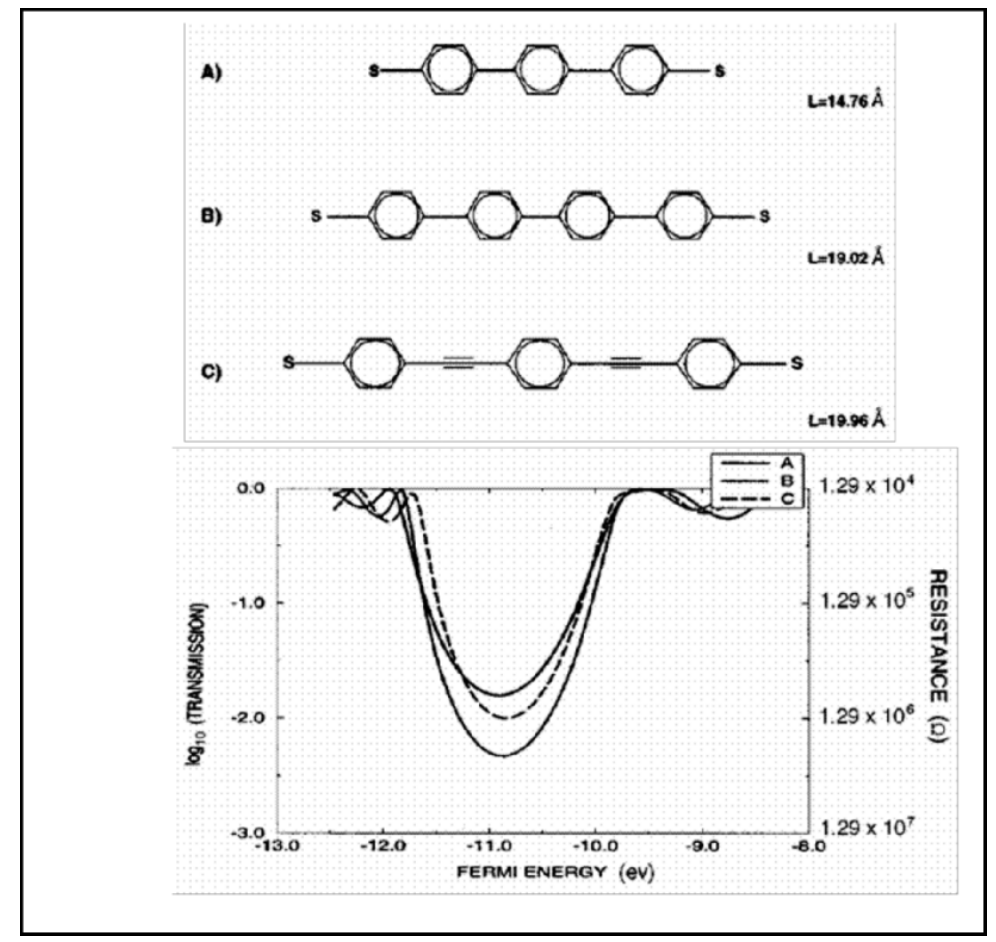

Fig. 3. Ring orientation effects on the transmission property. From Samanta, et al. [4], "Electronic conduction through organic molecules."

The growing body of theoretical work and tools used to generate molecular models has lead to many suggestions for novel, molecular devices. Shown in Figure 
4 are several examples of these devices, which include switches, wires, rectifiers and storage devices. Of course, the only way to determine the validity of theoretical models used to generate these suggestions is through experimental analysis, an area just now developing on its own. The contact to the molecule itself, atomic in nature, is just as much a part of the device as the organic structure itself. Although theoretical studies have helped to understand the nature of the metal-molecular contact, finding a consistent, scalable and repeatable test bed for comparing different molecules has proven to be the most challenging aspect of molecular characterization.

\section{Circuits and Scaling for Molecular Electronics}

It can be argued that to be a true successor technology to CMOS, molecular electronics needs to provide two of more generations of scaling beyond the silicon 18 $\mathrm{nm}$ node. That is provide continued scaling in some or more metrics of fundamental performance in computing, particularly computation throughput, power per operation, and cost per unit throughput; while achieving similar system availability and reliability rates to those achieved today.

These are challenging metrics to evaluate especially for an immature technology. This section will start off by reviewing the basics behind molecular electronics, and provide a summary evaluation of the potential, and roadblocks, for molecular electronics to provide continued scaling beyond the end of CMOS, in these metrics.

For reasons discussed above, all practical proposed and demonstrated molecular electronic devices are two terminal devices. For example, the illustration in Figure 5 is that of an atomic level presentation of benzene thiol molecules assembled between two gold contacts. The density potential of this technology is evident when it is realized that the molecule illustrated in Figure 5 is only $3.2 \mathrm{~nm}$ long. Generally speaking the principle of operation behind these devices is that a change in the longitudinal electric field causes a temporary or permanent change in the electron cloud configuration around the molecule and thus its conductivity. The molecule thus behaves like a tunneling diode. Measured results have shown characteristics that include non-rectifying diodes, rectifying diodes (by using different metals in the two contacts), diodes displaying negative differential resistance (NDR) and two-state (on-off) diodes, with an on-state and an off-state (Figure 6) [1]. Note that in Figure 6 only the rectifying version of the two-state diode is shown, as it is much more useful than the non-rectifying version. Of these characteristics devices, only the NDR and the rectifying on-off diodes are useful for logic, and even then present challenges over their 3-termimal predecessors.

In order to evaluate the potential of these devices, NAND gate configurations were compared with that of a $\sim 201818 \mathrm{~nm}$ node CMOS NAND gate. Figure 7 shows a possible circuit topology that uses the NDR diode to make a NAND gate and a circuit topology to build a programmable logic array using rectifying on-off diodes (only the on diodes are shown). Though the NDR-based circuit is impractical, it is included for completeness. The PLA structure requires a gain element to be practical. It is assumed that it is rebuffered using CMOS gates. 

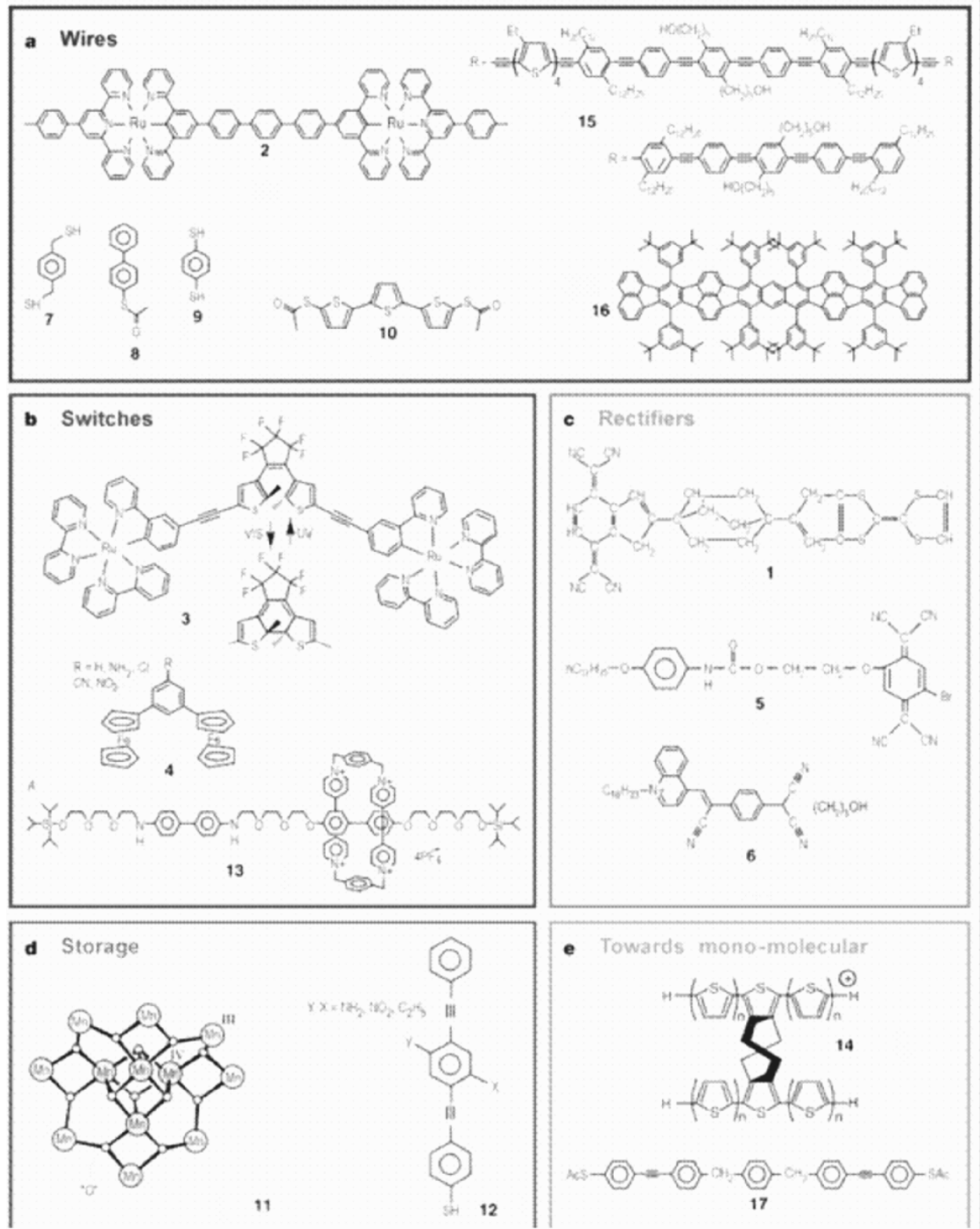

Fig. 4. Suggested molecular electronic devices. From Joachim et al. [6] "Electronics using hybrid-molecular and mono-molecular devices."

These three circuit topologies are evaluated against some useful performancerelated metrics in Table 1. The values for the $18 \mathrm{~nm}$ node NAND gate are taken from, or calculated using, data in the International Technology Roadmap for Semiconductors. The values for the molecular circuits are calculated using simple techniques and are likely to be wrong by several orders of magnitude. The area estimate for the PLA is made assuming an $8 \mathrm{~nm}$ wire imprint technology and 
(very pessimistically) and $2 \mathrm{x}$ area overhead for the peripheral circuits. The molecular device is assumed to have an on current of $500 \mathrm{nA}$ and an off current of 50 $\mathrm{nA}$. The delay and power estimates are made from circuit level calculations, not from the underlying physics.

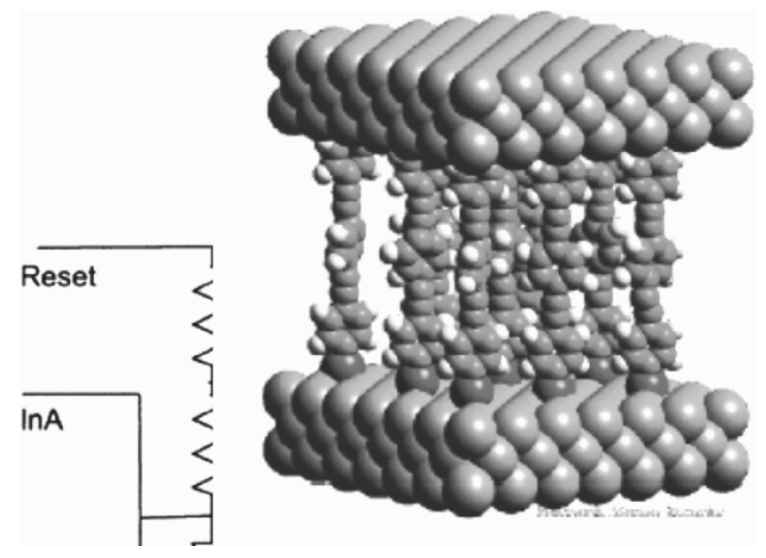

Fig. 5. Benzene Thiol molecules between two gold contacts. (Courtesy, Seminario).
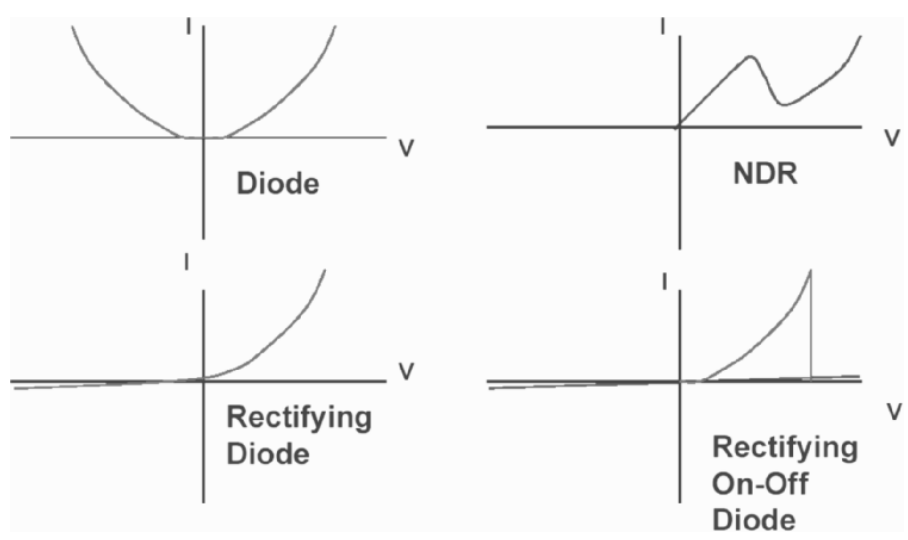

Fig. 6. Generic IV characteristics for molecular diodes. 


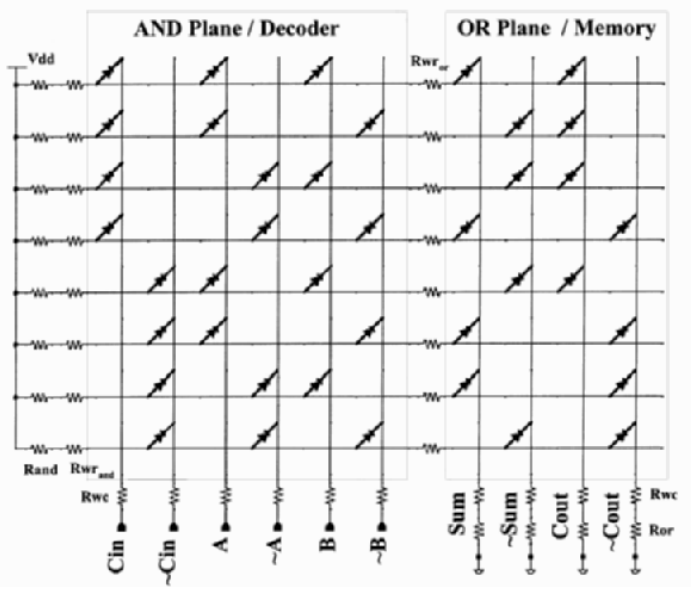

Fig. 7. Examples of circuits built using NDR (left side) and rectifying on-off diodes (right side). Source[7].

Table 1. Area and Performance Estimates for CMOS and molecular nanotechnologies.

\begin{tabular}{|l|c|c|c|c|}
\hline Circuit & Area & $\begin{array}{c}\text { Energy } \\
\text { per 010 } \\
\text { transition }\end{array}$ & $\begin{array}{c}\text { Leakage } \\
\text { Power }\end{array}$ & Delay \\
\hline $\begin{array}{l}18 \mathrm{~nm} \text { 2-input } \\
\text { NAND gate }\end{array}$ & $\begin{array}{c}0.2 \\
\text { sq. } \mu \mathrm{m}\end{array}$ & $10^{-17} \mathrm{~J}$ & $10^{-7} \mathrm{~W}$ & $5 \mathrm{ps}$ \\
\hline NDR gate & $\begin{array}{c}0.1 \\
\text { sq. } \mu \mathrm{m} ?\end{array}$ & $10^{-16} \mathrm{~J}$ & $10^{-12} \mathrm{~W}$ & $1 \mu \mathrm{s}$ \\
\hline $\begin{array}{l}\text { 2-input NAND } \\
\text { equivalent } \\
\text { within larger } \\
\text { PLA }\end{array}$ & $\begin{array}{c}0.01 \\
\text { sq. } \mu \mathrm{m} .\end{array}$ & $10^{-16} \mathrm{~J}$ & $10^{-7} \mathrm{~W}$ & $100 \mathrm{ps}$ \\
\hline
\end{tabular}

Unsurprisingly, given the use of two-terminal devices, the only aspect in which molecular electronics outperforms $18 \mathrm{~nm} \mathrm{CMOS} \mathrm{is} \mathrm{area.} \mathrm{Delay} \mathrm{is}$ significantly worse, and power comparable. The energy*delay product is worst in the molecular case, while the area*delay product is comparable.

This analysis leads to the tentative conclusion that most likely the best application for molecular electronics is in large relatively slow memories, and devices that benefit from such memories. Then the key question is what is the real density likely to be achieved by a molecular memory. There are two sub questions here - what is the peak density and what is the achievable density when peripheral circuits are accounted for? 
The peak density is related to the smallest wire that can be imprinted - likely to be around $5 \mathrm{~nm}$ wide. This gives a unit cell size of 10x10 nm, or a device density of 10,000 devices per sq. $\mu$ m., equivalent to a peak density of $10^{12}$ devices per sq.cm., or more than 10 full length DVD movies on a chip!

However, the achievable density is limited by the overhead required for address decoders, sense-amps, etc. This, in turn, is limited by the largest subarray that can be built and read with sufficiently low error rate. This has been analyzed and the results presented in Table 2 . The achievable sub-array size depends solely on the on:off ratio achieved by the diode. For reference, measured data seems to indicate on:off ratios today of around 10:1 for truly molecular devices. In contrast today's DRAMs are built using 10,000 x 10,000 subarrays. It is clear that larger on:off ratios are needed to achieve reasonable overheads. Fortunately, a number of nano-engineered device concepts are under investigation that has potential to achieve the required on:off ratio.

Table 2. The maximum sub-array size that can be built for different molecular diode on:off ratios.

\begin{tabular}{|l|l|}
\hline On:off Ratio & Max. Array \\
\hline \hline $7: 1$ & $64 \times 64$ \\
\hline \hline $13: 1$ & $128 \times 128$ \\
\hline \hline $100: 1$ & $1225 \times 1225$ \\
\hline \hline $1000: 1$ & $12 \mathrm{kx} 12 \mathrm{k}$ \\
\hline $8000: 1$ & $1 \mathrm{Mx} 1 \mathrm{M}$ \\
\hline
\end{tabular}

\section{Conclusions}

While molecular electronics holds significant potential, achieving that potential in a technologically useful fashion is very challenging. Challenges include the following. First there is the difficulty of integrating molecules with bulk materials in ways that the limitations of the latter do not dominate the device operation. This is why filament switching dominates many of the collected results. Second is achieving the challenge of achieving sufficient device performance such that molecules can outscale silicon in a metric beside size. However, with increased understanding of molecular design and performance, together with improving abilities to fabricate nano-ordered materials, molecular electronics is still a promising future technology. 


\section{References}

1. A. Aviram, M.A. Ratner, "Molecular rectifiers," Chem Phys. Lett., 29, 277, 1974.

2. C. Joachim, J.K. Gimzewski, A. Aviram, "Electronics using hybrid-molecular and mono-molecular devices," Nature, 408, 541-548, 2000.

3. Robert W. Keyes, "Fundamental Limits of Silicon Technology," Proc. IEEE, 89, 227-239, 2001.

4. M.P. Samanta, W. Tian, S. Datta, J.I. Henderson, C.P. Kubiak, "Electronic conduction through organic molecules," Phys. Rev. B, 53, 7626-7629, 1999.

5. Prashant Damle, Titash Rakshit, Magnus Paulsson, Supriyo Datta, "CurrentVoltage Characteristics of Molecular Conductors: Two Versus Three Terminal," IEEE Nano, 1, 145-153, 2002.

6. C. Joachim, J.K. Gimzewski, A. Aviram, "Electronics using hybrid-molecular and mono-molecular devices," Nature, 408, 541-548, 2000.

7. M.R. Stan, P.D. Franzon, S.C. Goldstein, J.C. Lach, M. Zigler, "Molecular Electronics: from devices and interconnect to circuits and architecture," Proc. IEEE, 91(11), Nov. 2003, pp. 194-1957. 